\title{
Early detection of active glomerular lesions in dogs and cats using podocin
}

\author{
Barbara Szczepankiewicz ${ }^{1}$, Urszula Pasławska ${ }^{1,2}$, Marcin Nowak ${ }^{3}$, Remigiusz Bąchor ${ }^{4}$, \\ Agnieszka Czyżewska-Buczyńska ${ }^{5}$, Robert Pasławski ${ }^{2}, Z^{2}$ igniew Szewczuk ${ }^{4}$ \\ ${ }^{1}$ Department of Internal Medicine and Clinic of Diseases of Horses, Dogs and Cats, Faculty of Veterinary Medicine, Wrocław \\ University of Environmental and Life Sciences, 50-366 Wrocław \\ ${ }^{2}$ Institute of Veterinary Medicine, Faculty of Biological and Veterinary Sciences, \\ Nicolaus Copernicus University Toruń, 87-100 Toruń \\ ${ }^{3}$ Department of Pathology, Faculty of Veterinary Medicine, \\ Wrocław University of Environmental and Life Sciences, 50-375 Wrocław \\ ${ }^{4}$ Department of Chemistry, Wrocław University, 50-383 Wrocław \\ ${ }^{5}$ Regional Specialist Hospital in Wrocław, Research and Development Unit, 51-124 Wrocław \\ barbara.szczepankiewicz@upwr.edu.pl
}

Received: July 29, $2019 \quad$ Accepted: November 5, 2019

\begin{abstract}
In veterinary medicine, sensitive and specific markers of the early stages of renal failure still remain to be established. Podocytes could be a promising diagnostic tool in veterinary nephrology, especially in the differentiation of active pathological disease and glomerulopathies. Podocin is one of the robust proteins exploitable in detection of podocyturia. This article presents podocyte detection in urine for diagnostic purposes in veterinary medicine using a variety of methods. We describe the advantages and disadvantages of the immunohistochemical technique currently used, and of scanning microscopy, chromatography, and immunostaining. The identification of podocin-positive cells is a promising diagnostic tool in the detection of the early stages of glomerular basement membrane damage. The detection of renal failure prior to the occurrence of azotaemia is of high clinical importance from the clinical and scientific points of view.
\end{abstract}

Keywords: podocytes, podocyturia, urinalysis, glomerulopathy, kidneys, markers.

\section{Introduction}

Chronic kidney disease (CKD) is a major cause of morbidity and mortality in dogs and cats. The prevalence of CKD has been estimated to be $0.5-1.0 \%$ in dogs and $1.0-3.0 \%$ in cats. Epidemiological studies show that the incidence of CKD increases with age, especially in cats. Nephron damage associated with CKD is usually irreversible and often progressive. The disease affects $10-25 \%$ of dogs and cats $>10$ years old in referral institutions and $30-50 \%$ of cats 15 years of age or older $(10,14,23)$. In human medicine the lower limits of the ranges are higher with $11.7-15.11 \%$ of people suffering from CKD and $35 \%$ of people over 70 years old (12). This discrepancy suggests that the prevalence in veterinary medicine is underestimated because of a lack of known sensitive and specific markers of the early stages of renal injury. Since the inception of the
International Renal Interest Society (IRIS) staging of CKD and acute kidney injury (AKI) (14) grading systems to categorise and stratify kidney disease in animals, there has been confusion over and misunderstanding of the value and clinical utility of the early (non-azotaemic) categories described there (8). Podocytes can be a potentially helpful tool in diagnosing kidney injury at the beginning of the illness.

Podocytes are a key element from the point of view of selective plasma filtration and primary urine production, because they build the visceral lamina of the glomerular capsule (24). Long primary and secondary processes, also known as foot processes or pedicels, exit the podocyte cell body. The primary process of podocytes contains podocin, a $42 \mathrm{kDa}$ protein (2). Podocin binds to the cytoplasmic part of nephrin and $\mathrm{CD} 2 \mathrm{AP}$ protein and together with them maintains important podocyte functions, i.e. survival, 
proliferation, differentiation, and construction of the cytoskeleton $(14,24)$. Pathological processes that occur in the kidneys cause podocyte tearing and excretion, elevating the urinary podocyte content $(11,30)$. Podocytes being non regenerative, their loss is irreversible (45). It has been shown that a $20-40 \%$ loss of glomerular podocytes, i.e. approximately 100-200 podocytes per glomerulus, leads to glomerulosclerosis and kidney function decline (21).

Numerous reports indicate that an early diagnosis of an elevated urinary podocyte excretion may facilitate the diagnosis of kidney diseases in humans and animals $(4,30)$. In addition, the loss of podocytes is a sustained process (self-expanding process); because the lost podocytes do not regenerate, the remaining podocytes must be enlarged to cover the basement membrane, and this mechanism leads to irreversible scarring of the glomerulus (6). Several studies reported that loss of podocytes (podocytopenia) is correlated with the development of glomerulopathy (28).

The podocin-positive cells detected in humans with glomerulonephritis included not only podocytes of the glomerular basement membrane, but also parietal glomerular epithelial cells (PEC) and proximal tubule epithelial cells (PTEC) (1). However, the majority of podocin-positive cells were podocytes rather than PEC or PTEC (1). Moreover podocyturia correlated in humans with a reduction of glomerular filtration rate and renal failure (16). The most interesting finding was the discovery that podocyturia correlated positively with the active inflammation process - hence it is a unique marker that allows active kidney disease processes to be distinguished from inactive ones $(11,19,31)$. Proteinuria, unlike podocyturia, occurs in both active kidney disease and in its chronic form, and is therefore a less sensitive marker of kidney disease processes (14).

Podocyte testing may be based on their determination using a commercial test. The advantages of the test are easy accessibility and cost-effectiveness. The antibodies detected in ELISA may be a useful tool in the diagnosis of acute and chronic glomerular failure in dogs, as well as in monitoring the progression or regression of the disease. In addition, podocytes may be useful in the diagnosis of renal or non-renal proteinuria. While the podocyte number depends on concentration of urine, the results should be correlated with urinary creatinine in order to assess urine density (especially in patients with polyuria and polydipsia).

Podocin (rabbit anti-human podocin antibody, Sigma-Aldrich, USA.) was also used to identify podocytes in the histopathology probe of dog kidneys (13, 17, 27) (Fig. 1).

Canine-specific podocin sequences were found that could potentially be used for the production of laboratory tests for canine-specific primers for podocyte gene products (9) and canine podocin ELISA (MyBioSource, USA) and canine antibodies (Abexxa, UK) (Fig. 1). The assessment of the number of podocytes in histopathological preparations is difficult and does not seem to be very practical in clinical settings.
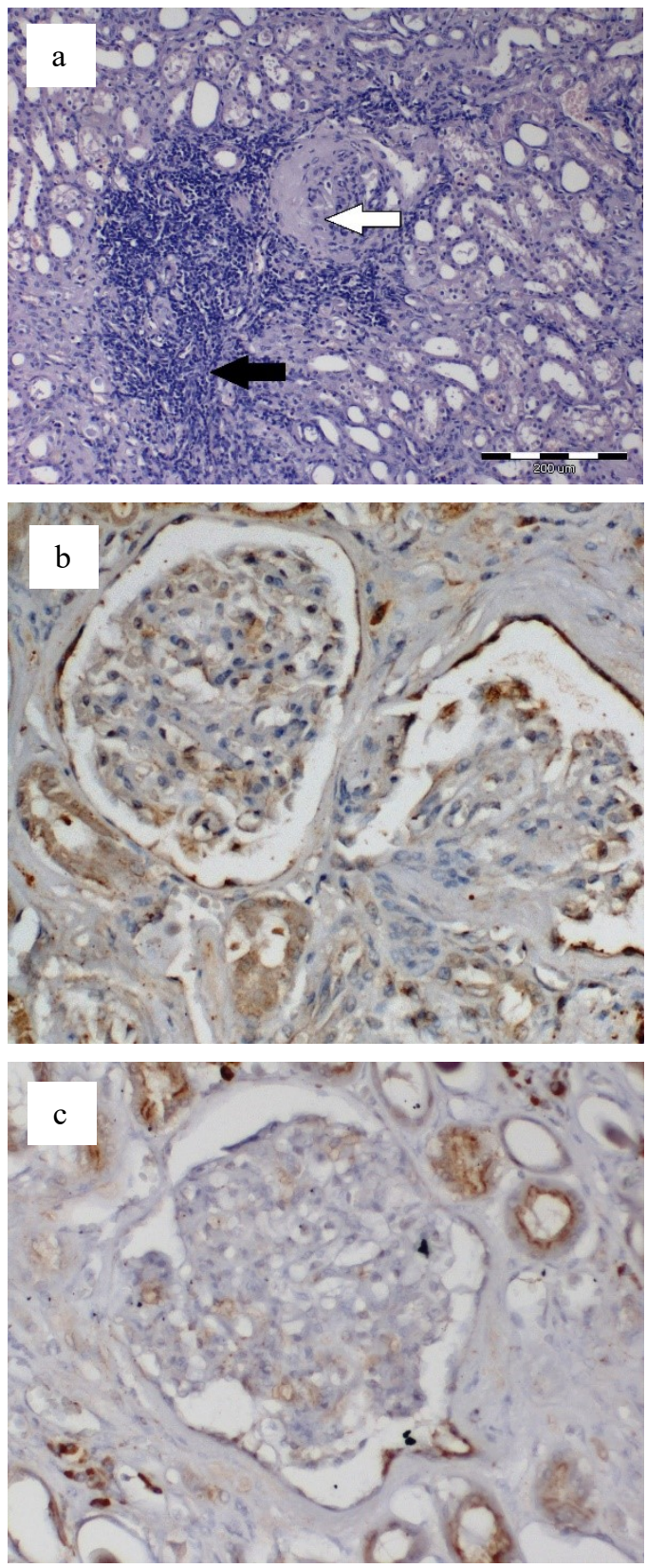

Fig. 1. A histopathological assessment of the kidney of a dog without blood azotaemia, suffering from cardiac insufficiency due to severe stage Da mitral endocardiosis according to the ACVIM classification (9)

a - H-E stained section. Interstitial, lymphocytic inflammatory infiltration (black arrow) and glomerular hyalinosis and atrophy (white arrow) are visible;

$\mathrm{b}$ - the immunohistochemical reaction in the kidney section of the same dog, using polyclonal rabbit anti-nephrin antibody (cat no. abx019258, Abexxa, UK, 1:200 dilution). A weak reaction indicates podocyte damage;

c - a weak immunohistochemical reaction indicating podocyte damage, using polyclonal rabbit anti-podocin antibody (cat no. abx019257, Abexxa, 1:400 dilution). The sections were washed with Dako Wash Buffer $(10 \times)$ (product code S3006, Agilent, USA). The Peroxidase/DAB + Rabbit/Mouse Dako Real EnVision Detection System was used to visualise the immunohistochemical reaction (product code K8006, Agilent). The microphotographs of the studied sections come from our own research and were subjected to computeraided image analysis using cellSens software (Olympus Soft Imaging Solution GmbH, Germany) coupled with an optical Olympus BX53 microscope equipped with a ColorView IIIu digital camera (Olympus, Japan) 


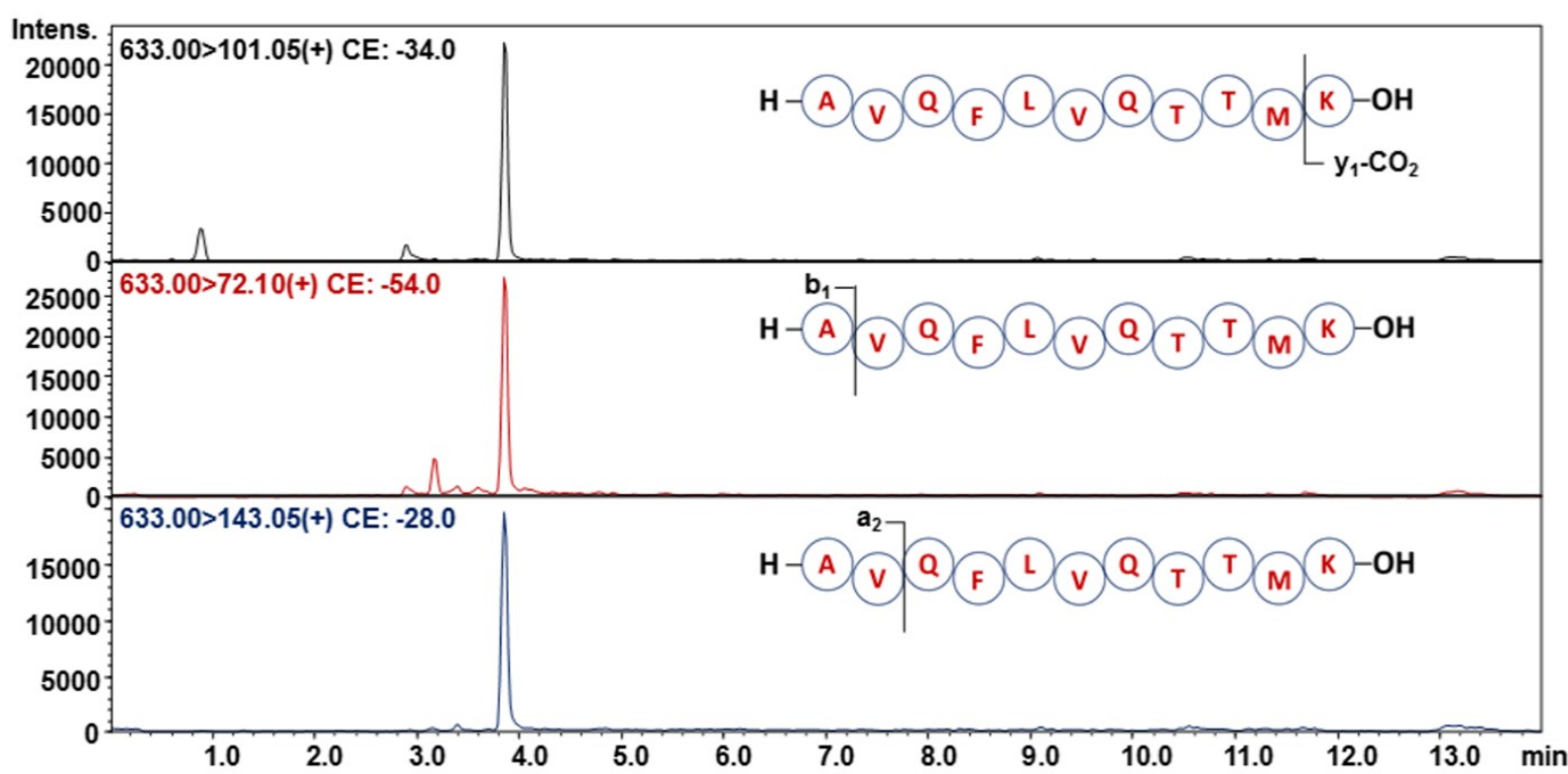

Fig. 2. Multiple reaction monitoring (MRM) chromatograms for the analysis of an H-AAEILAATPAAVQLR-OH peptide, which is a fragment of canine podocin in urine sediment. The retention time of $3.78 \mathrm{~min}$ corresponds to the studied compound. The presence of an additional signal at 3.32 min is associated with the presence of cis-trans isomerism of the proline residues (29)

In general, urine examination allows the detection of kidney damage prior to the occurrence of clinical signs, the assessment of the degree of kidney damage, and the identification of the pathological processes occurring in the kidneys, without the need for a kidney biopsy. Owners of animals suffering from or suspected of having kidney diseases are usually very reluctant to give consent to a veterinarian's anaesthetising the patient and performing a kidney biopsy. Their concerns are related to the possible complications of this invasive procedure. Very rarely, histopathological examination is performed from a removed kidney, because it is not a popular method in veterinary medicine. Moreover, some patients have contraindications for kidney biopsy (22).

A promising method to determine podocytes of canine and feline urine and tissue samples seemed to be real-time reverse transcription PCR. Podocin was assessed by RT-PCR examination using the canine NPHS2 XM_547443 forward primer 5'GCCCAAGATCTAAAGGTTGC-3' and reverse counterpart 5'-ACAGCCAGTGAGTGCTGAAG-3' (predicted size $116 \mathrm{bp}$ ) and feline forward and reverse primers 5'-CTGTGAGTGGCTTCTTGTCCTC-3' and 5'-GGAAGCAGATGTCCCAGTCG-3' (predicted size 132 bp). The result was disappointing. There was no difference in podocin expression in urine samples or in the kidney tissue taken from healthy dogs and dogs suffering from kidney disease (13).

Another method for recognition of podocytes using podocin was liquid chromatography coupled with mass spectrometry (LC-MS). It has recently become the method of choice in the analysis of protein mixtures in urine due to its sensitivity and accuracy, making the analysis of complex biological samples possible (20, 25). Identification of compounds was based on molecular masses of protein biomarkers and their fragments obtained in tandem mass spectrometry (MS/MS) or multiple reaction monitoring (MRM mode).

The major advantage of spectrometry in protein biomarker investigation in comparison to the immunochemical methods is the possibility of analysis of many biomarkers in one experiment. Additionally, there is no need for antibody generation. Moreover, direct analysis of known compounds using MRM mode provides higher sensitivity and selectivity and allows fine-tuning of an instrument to search for the compound of interest and its fragment ion (26). This approach allows for the detection of low-abundance molecules in highly complex mixtures. Additionally, targeted analysis using MRM mass spectrometry enhances the detection limit for peptides and allows continuous and fast monitoring of the specific ions of interest. The unquestionable advantages of the MRM method are its time-effectiveness, complete procedure automation, high sensitivity, precision, and repeatability (3). Multiple reaction monitoring (MRM) chromatograms for the analysis of an H-AAEILAATPAAVQLR-OH peptide, which is a fragment of canine podocin in urine sediment, are presented in Fig. 2 (29).

However, the basic limitation of this method is the availability of MRM. At the time of writing, it seems that the small number of these devices and the cost of the test may be limiting factors on the method's use in practice. The results in humans show some minor physiological podocyturia (31). It is not clear if this phenomenon exists in dogs and cats, or if it is present, what its impact on the MRM results is.

Podocytes may be a useful tool in the diagnosis of acute and chronic glomerular failure in dogs, as well as in monitoring the progression or regression of the disease. In addition, podocytes may be exploitable in the diagnosis of renal or non-renal proteinuria. Accurate 
quantification of changes in the number of podocytes is recommended, however, no specific and useful marker has been identified to date that enables a podocyte count (5).

Previous studies indicate that podocytes are less useful in diagnosing kidney disease in cats than in dogs. This is related to the species-specificity of the particular kidney disease - primary glomerular disease was detected very seldom in cats, because most with chronic kidney disease had tubulointerstitial pathologies (14). Lesions of glomerulosclerosis were relatively mild and overshadowed by tubulointerstitial lesions (6). In contrast to cats, most dogs suffer from glomerular injury (7), and glomerular disease is estimated to afflict about $52 \%$ of dogs (18). In veterinary medicine, primary glomerulopathies are defined as those nephropathies that arise with involvement of glomeruli in the processes that initiate renal injury, regardless of their pathologic mechanisms, whereas secondary glomerular changes occur after renal tubular or whole nephron damage. In contrast, in human medicine, the term "primary glomerular disease" connotes disease which primarily involves the kidney and "secondary glomerular disease" means a disease in which kidney involvement is part of a systemic disorder (14).

Despite all doubts, the diagnostic value of podocytes in veterinary medicine is promising, especially in the context of differentiation of the active and inactive forms of glomerular injury. Each biomarker of active glomerular injury has the potential to augment the canon of knowledge of chronic kidney disease, as a possible means for its early identification and mediator of its progression. The detection of renal failure prior to the occurrence of azotaemia is of high clinical importance, as the clinical signs of renal failure, such as elevated levels of serum urea and creatinine are present only at an advanced stage of kidney damage. The implementation of podocyturia testing in routine dog and cat urinalysis may reveal asymptomatic patients at an early stage of CKD or AKI. The apparent difference in the incidence of CKD and AKI between humans and pets may partly be caused by an underestimation of CKD due to inaccurate diagnosis of early kidney damage. Podocyturia may be the first detectable indicator in dogs and cats of kidney failure due to damage of the glomerular basement membrane.

Conflict of Interests Statement: The authors declare that there is no conflict of interests regarding the publication of this article.

Financial Disclosure Statement: This article was supported by grant no. UMO-2016/23/B/ST4/01036 from the National Science Centre, Poland.

Animal Rights Statement: None required.

\section{References}

1. Achenbach J., Mengel M., Tossidou I., Peters I., Park J.K., Haubitz M., Ehrich J.H., Haller H., Schiffer M.: Parietal epithelia cells in the urine as a marker of disease activity in glomerular diseases. Nephrol Dial Transplant 2008, 23, 3138-3145.

2. Arif E., Nihalani D.: Glomerular Filtration Barrier Assembly: An insight. Postdoc J 2013, 1, 33-45.

3. Bąchor R., Mielczarek P., Rudowska M., Silberring J., Szewczuk Z.: Sensitive detection of charge peptides at the attomole level using nano-LC-ESI-MRM analysis. Int J Mass Spectrom 2014, 362, 32-38.

4. Bąchor R., Szczepankiewicz B., Pasławska U., Mojsa K., Stefanowicz P., Szewczuk Z.: Detection of tryptic podocin peptide in the feline urine samples using LC-MS/MRM method. Int J Mass Spectrom 2019, 444, 116174. doi: 10.1016/j.ijms.2019.116174.

5. Camici M.: Urinary detection of podocyte injury. Biomed Pharmacother 2007, 61, 245-249.

6. Chakrabarti S., Syme H.M., Brown C.A., Elliott J.: Histomorphometry of feline chronic kidney disease and correlation with markers of renal dysfunction. Vet Pathol 2013, 50, 147-155

7. Cianciol R.E., Mohr F.C., Aresu L., Brown C.A., James C., Jansen J.H., Spangler W.L., van der Lugt J.J., Kass P.H., Brovida C., Cowgill L.D., Heiene R., Polzin D.J., Syme H., Vaden S.L., van Dongen A.M., Lees G.E.: World Small Animal Veterinary Association Renal Pathology Initiative: classification of glomerular diseases in dogs. Vet Pathol 2016, 53, 113-135.

8. Cowgill L.D., Polzin D.J., Elliott J., Nabity M.B., Segev G., Grauer G.F., Brown S., Langston C., van Dongen A.M.: Is progressive chronic kidney disease a slow acute kidney injury? Vet Clin North Am Small Anim Pract 2016, 46, 995-1013.

9. Farooqi E.: Development of a novel diagnostic test using podocyturia as a biomarker for detection of kidney damage. Bachelor of Science thesis .Texas A\&M University 2013.

10. Finch N.C., Syme H.M., Elliott J.: Risk factors for development of chronic kidney disease in cats. J Vet Intern Med 2016, 3, 602-610.

11. Hara M., Yanagihara T., Hirayama Y., Ogasawara S., Kurosawa H., Sekine S., Kihara I.: Podocyte membrane vesicles in urine originate from tip vesiculation of podocyte microvilli. Hum Pathol 2010, 41, 1265-1275.

12. Hill N.R., Fatoba S.T., Oke J.L., Hirst J.A., O’Callaghan C.A., Lasserson D.S., Hobbs F.D.: Global prevalence of chronic kidney disease - a systematic review and meta-analysis. PLoS One 2016, 11, e0158765. doi: 10.1371/journal.pone.0158765.

13. Ichii O., Yabuki A., Sasaki N., Otsuka S., Ohta H., Yamasaki M., Takiguchi M., Namiki Y., Hashimoto Y., Endoh D., Kon Y.: Pathological correlations between podocyte injuries and renal functions in canine and feline chronic kidney diseases. Histol Histopathol 2011, 26, 1243-1255.

14. http://www.iris-kidney.com/guidelines/staging.html.

15. Jalanko H.: Pathogenesis of proteinuria: lessons learned from nephrin and podocin. Pediatr Nephrol 2003, 18, 487-491.

16. Kikuchi M., Wickman L., Rabah R., Wiggins R.C.: Podocyte number and density changes during early human life. Pediatr Nephrol 2017, 32, 823-834.

17. Kobayashi R., Kamiie J., Yasuno K., Ogihara K., Shirota K.: Expression of nephrin, podocin, $\alpha$-actinin- 4 and $\alpha 3$-integrin in canine renal glomeruli. J Comp Pathol 2011, 145, 220-225.

18. MacDougall D.F., Cook T., Steward A.P., Cattell V.: Canine chronic renal disease: prevalence and types of glomerulonephritis in the dog. Kidney Int 1986, 29, 1144-1151.

19. Nakamura T., Ushiyama C., Suzuki S., Hara M., Shimada N., Ebihara I., Koide H.: The urinary podocyte as a marker for the differential diagnosis of idiopathic focal glomerulosclerosis and minimal-change nephrotic syndrome. Am J Nephrol 2000, 20, 175-179. 
20. Panek-Laszczyńska K., Konieczny A., Milewska E., Dąbrowska K., Bąchor R., Witkiewicz W., Szewczuk Z.: Podocyturia as an early diagnostic marker of preeclampsia: a literature review. Biomarkers 2018, 23, 207-212.

21. Puelles V.G., Bertram J.F.: Counting glomeruli and podocytes: rationale and methodologies. Curr Opin Nephrol Hypertens 2015, 24, 224-230.

22. Rawlings C.A., Diamond H., Howerth E.W., Neuwirth L., Canalis C.: Diagnostic quality of percutaneous kidney biopsy specimens obtained with laparoscopy versus ultrasound guidance in dogs. J Am Vet Med Assoc 2003, 1, 317-321.

23. Rudinsky A.J., Harjes L.M., Byron J., Chew D.J., Toribio R.E., Langston C., Parker V.J.: Factors associated with survival in dogs with chronic kidney disease. J Vet Intern Med 2018, 32, 1977-1982.

24. Scott R.P., Quaggin S.E.: The cell biology of renal filtration. J Cell Biol 2015, 27, 199-210.

25. Seidler J., Zinn N., Boehm M.E., Lehmann W.D.: De novo sequencing of peptides by MS/MS. Proteomics 2010, 10, 634-649.

26. Simon R., Lemoine J., Fonbonne C., Jaffuel A., Léonard J.F., Gautier J.C., Pasquier O., Salvador A.: Absolute quantification of podocin, a potential biomarker of glomerular injury in human urine, by liquid chromatography-multiple reaction monitoring cubed mass spectrometry. J Pharm Biomed Anal 2014, 9, 84-91.
27. Sugahara G., Kamiie J., Kobayashi R., Mineshige T., Shirota K.: Expression of phospholipase A2 receptor in primary cultured podocytes derived from dog kidneys. J Vet Med Sci 2016, 1, 895-899.

28. Suzuki T., Matsusaka T., Nakayama M., Asano T., Watanabe T., Ichikawa I., Nagata M.: Genetic podocyte lineage reveals progressive podocytopenia with parietal cell hyperplasia in a murine model of cellular/collapsing focal segmental glomerulosclerosis. Am J Pathol 2009, 174, 1675-1682.

29. Szczepankiewicz B., Bąchor R., Pasławski R., Siwińska N., Pasławska U., Konieczny A., Szewczuk Z.: Evaluation of tryptic podocin peptide in urine sediment using LC-MS-MRM method as a potential biomarker of glomerular injury in dogs with clinical signs of renal and cardiac disorders. Molecules 2019, 24, 3088. doi: 10.3390/molecules24173088.

30. Szczepankiewicz B., Pasławska U., Paslawski R., Gebarowski T., Zasada W., Michalek M., Noszczyk-Nowak A.: The urine podocin/creatinine ratio as a novel biomarker of cardiorenal syndrome in dogs due to degenerative valve disease. J Physiol Pharmacol 2019, 70, doi: 10.26402/jpp.2019.2.06.

31. Vogelmann S.U., Nelson W.J., Myers B.D., Lemley K.V.: Urinary excretion of viable podocytes in health and renal disease. Am J Physiol Renal Physiol 2003, 285, 40-48. 